\title{
Impurity Transport Study of Medium-Z Argon by Means of Soft $X$-Ray Pulse Height Analyzer in LHD
}

\author{
Sadatsugu MUTO, Shigeru MORITA and LHD Experimental Group \\ National Institute for Fusion Science, Toki 509-5292, Japan
}

(Received 19 December 2006 / Accepted 6 April 2007)

\begin{abstract}
Radial profiles of impurity transport coefficients of argon have been successfully obtained in Large Helical Device, using an assembly equipped with conventional semiconductor detectors and soft x-ray pulse-height analyzers. Several fixed argon discharges have enabled the radial scanning of the assembly to measure the radial profiles of argon- $\mathrm{K}_{\alpha}$ lines. The present experimental results indicate that the impurity transport study becomes possible with the soft x-ray pulse-height analyzers.
\end{abstract}

(c) 2007 The Japan Society of Plasma Science and Nuclear Fusion Research

Keywords: argon, impurity transport, particle diffusion, convective velocity, Large Helical Device

DOI: $10.1585 /$ prr.2.S1069

\section{Introduction}

Measurement of x-ray energy spectrum is important to obtain significant information from core plasma such as electron temperature, heavy impurity concentration, impurity transport coefficients and non-thermal electron density [1]. Especially, the measurement of space- and timeresolved x-ray energy spectra are essentially required in terms of the impurity transport study. In order to obtain such spectra, only a few diagnostics have been constructed until now. In JET tokamak a diffusion coefficient has been evaluated combining with an impurity injection [2].

Signals obtained with an x-ray detector are line integrated information along the line-of-sight of the detector. Then, an inversion process is necessary to the signal analysis in order to obtain a local emissivity. The difficulty of the process strongly depends on the relation between the structure of plasma magnetic surface and the line-of-sight. In order to eliminate a large error after inversion, the $\mathrm{x}$-ray detector installed in Large Helical Device (LHD; $R_{a x}=3.6 \mathrm{~m}, a=0.64 \mathrm{~m}, B=2.8 \mathrm{~T}$ ) has been set at the vertically-elongated plasma cross-section and measured perpendicularly to the magnetic axis from the bottom of the plasma [3].

As a general method for studying the impurity transport, an impurity element is injected into the plasma $[4,5]$. However, the decreasing of electron temperature due to the relatively much amount of impurity injection often gives rise to a non negligible perturbation to the transport. It is the most important advantage of the x-ray diagnostics that the intensity of $x$-ray lines is enough to investigate the impurity transport, even if the metallic impurity density is 4 orders less than the electron density [6]. Consequently, $x-$ ray measurement is one of the best methods for reducing the amount of impurity injection. In an analysis on the im-

author'se-mail: mutos@ms.nifs.ac.jp purity transport the strong temperature dependence of the $\mathrm{X}$-ray emissivity has to be taken into account.

In this paper experimental results in LHD are presented on time-resolved $\mathrm{Ar}-\mathrm{K}_{\alpha}$ profiles observed with an assembly equipped with semiconductor detectors and $\mathrm{x}$ ray pulse-height analyzers (PHA). Furthermore, the analysis on the impurity transport concerning the radial profiles of diffusion coefficient and convective velocity is also reported.

\section{Assembly for X-Ray Measurement}

The measurement of time-resolved profiles of x-ray lines emitted from highly ionized argon has been carried out in LHD using the assembly equipped with PHA and a movable slit which can scan the line-of-sight along the major radius direction. The performance of the assembly has been reported with observed $\mathrm{K}_{\alpha}$ lines of metallic impurities and continuum in a reference [7]. It must be mentioned here that the time- and space-resolutions are not simultaneously obtained with the assembly. In order to obtain many radial points required for a good radial profile, several fixed discharges have been used for the measurement.

The $\mathrm{x}$-ray intensity obtained with the assembly is lineintegrated signal along the line-of-sight. In order to analyze the radial profile of the emissivity, the integration equation is derived from the arrangement of the assembly as follows;

$$
F(\rho)=2 \int_{0}^{\infty} \mathrm{d} z f(c),
$$

where $\rho, F(\rho)$ and $f(\rho)$ are the normalized radius, the intensity profile observed with the assembly, and the emissivity profile, respectively. Of course, it is assumed that the $\mathrm{X}$-ray intensity emitted from impurities is constant along the magnetic surface. A sign " $c$ " in Eq. (1) means a mag- 
netic surface which crosses the line-of-sight at a position of $z$. In the present research the surface is approximately evaluated from an ellipse defined with an equation of

$$
\left(\frac{\rho-\varepsilon c}{c}\right)^{2}+\left(\frac{z}{\gamma c}\right)^{2}=1
$$

where $\varepsilon$ and $\gamma$ are constant values. The absolute value of $\varepsilon$ is less than 1 . Then, $c$ is solved as follows;

$$
c=\left(1-\varepsilon^{2}\right)^{-1}\left(-\varepsilon \rho+\sqrt{\rho^{2}+\frac{1-\varepsilon^{2}}{\gamma^{2}} z^{2}}\right) .
$$

From Eq. (1) and Eq. (3) the emissivity profile is mathematically solved as follows;

$$
\begin{aligned}
f(\rho)= & \left(2 \pi^{\frac{3}{2}} \gamma i\right)^{-1} \int_{b-i \infty}^{b+i \infty} \mathrm{d} s\left(1-\varepsilon^{2}\right)^{-s+\frac{1}{2}} \\
& \times \Theta^{-1}(s) g(s) \rho^{-s},
\end{aligned}
$$

where $i$ and $b$ are a complex sign and a constant real number, respectively. A function of $\Theta(s)$ in Eq. (4) is defined by

$$
\begin{aligned}
\because \Theta(s) \equiv & \sum_{n=0}^{\infty} \frac{(-\varepsilon)^{n}}{n !} s(s-1) \bullet \cdot(s-n+1) \\
& \times \frac{\Gamma\left(\frac{-s+n-1}{2}\right)}{\Gamma\left(\frac{-s+n}{2}\right)},
\end{aligned}
$$

where $\Gamma$ is a gamma function. A function of $g(s)$ is defined by

$$
\because g(s) \equiv \int_{0}^{\infty} \mathrm{d} z F(z) z^{s-2}
$$

Then, it becomes possible to invert the line-integrated $\mathrm{x}$ ray signal to the emissivity profile through the calculation process expressed in Eq. (4).

\section{Experimental Results}

An example of x-ray spectra obtained in the present experiment is shown in Fig. 1 . The $\mathrm{K}_{\alpha}$ line of argon appears at a photon energy of $3.2 \mathrm{keV}$. Taking into account the transmission rate of a beryllium filter with a thickness of 1 $\mathrm{mm}$, the spectrum has to be modified by a dashed curve in Fig. 1. Then, the real intensity of the $\operatorname{argon} \mathrm{K}_{\alpha}$ is 2 orders stronger than the metallic $\mathrm{K}_{\alpha}$ lines. The continuum intensity is negligible in comparison with the strong argon line at $3.2 \mathrm{keV}$. Accordingly, the radiation loss is dominated by the $\mathrm{K}_{\alpha}$ emission from $\mathrm{He}$ - and $\mathrm{H}$-like argon [8].

Figure 2 shows the emissivity profiles of $\mathrm{Ar}-\mathrm{K}_{\alpha}$ line obtained from the experimental result using Eq. (4). The profiles are smoothed, since the number of the line-of-sight is limited to $15(=3 \times 5)$ in the radial location. Here, the assembly has 3 line-of-sights and then 5 fixed discharges are

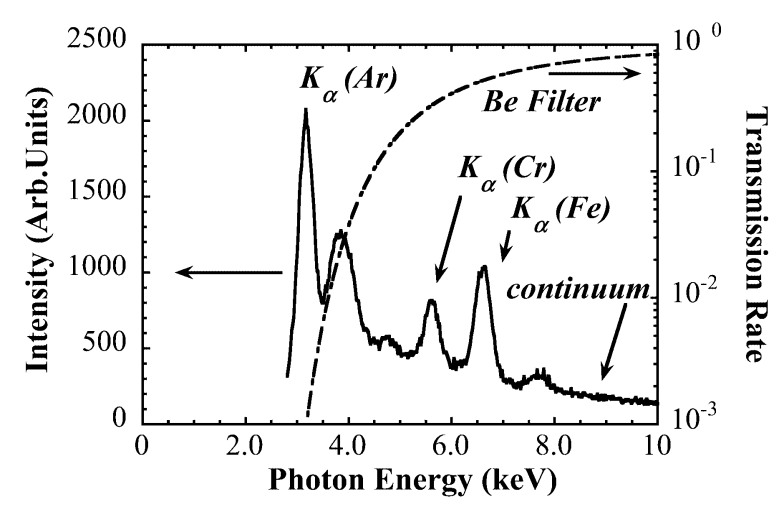

Fig. 1 Typical x-ray energy spectrum obtained from LHD NBI discharges with argon puff (solid line). $\mathrm{K}_{\alpha}$ emissions from argon, chromium and iron appear at $3.2 \mathrm{keV}$, $5.6 \mathrm{keV}$ and $6.6 \mathrm{keV}$, respectively. Transmission rate of beryllium filter is also indicated with dashed line.

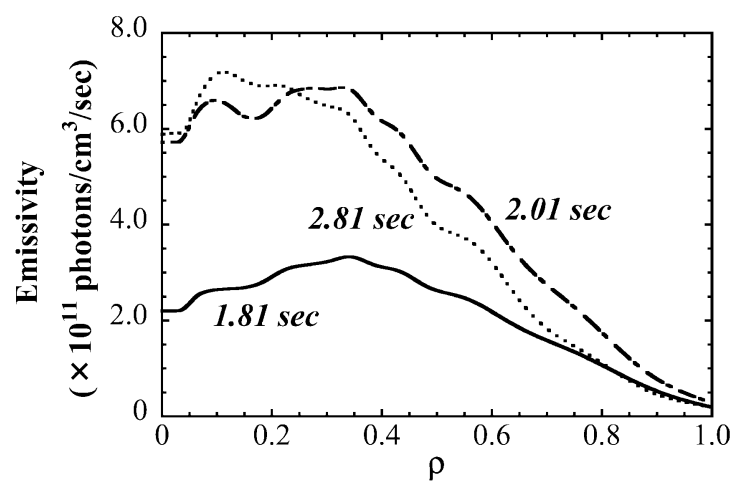

Fig. 2 Radial emissivity profiles of $\mathrm{Ar}-\mathrm{K}_{\alpha}$ line obtained using Eq. (4) at $1.81 \mathrm{~s}$ (solid line), $2.01 \mathrm{~s}$ (dashed line) and $2.81 \mathrm{~s}$ (dotted line), respectively as a function of the normalized radius. Argon gas is puffed at $1.50 \mathrm{~s}$.

needed for the 15 radial points observation. In the present experiment the profile has been measured with a time resolution of $0.02 \mathrm{~s}$. In the figure the emissivity profiles after inversion are indicated at $t=1.81 \mathrm{~s}, 2.01 \mathrm{~s}$ and $2.81 \mathrm{~s}$. The argon puff is done at $t=1.50 \mathrm{~s}$. It is clear that the penetration of the argon to the plasma core needs roughly $0.5 \mathrm{~s}$. The argon emissivity gradually reduces from the outside of the plasma due to the particle diffusion process.

Figure 3 shows the time evolution of $\mathrm{Ar}-\mathrm{K}_{\alpha}$ emissivity obtained with the assembly. Electron temperature profile, which is measured by Thomson scattering diagnostics, has been maintained to be constant in the fixed discharges. The time evolution of the central electron temperature is also shown in Fig. 3. Consequently, the emissivity approximately reflects only the amount of argon. The amount of argon rapidly increases after the argon puff. A phase shift among the x-ray emissivities is clearly obtained in the duration from $1.50 \mathrm{~s}$ to $2.00 \mathrm{~s}$ depending on the radial location as is shown in the figure. Qualitatively, the rising time of 


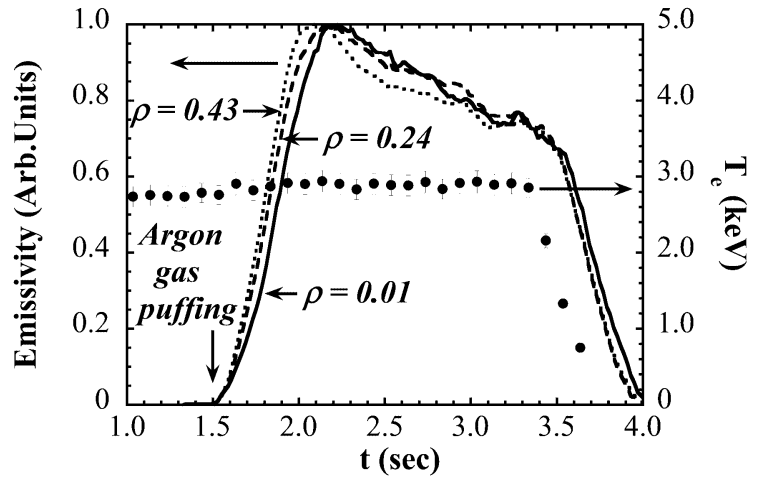

Fig. 3 Emissivities of argon $\mathrm{K}_{\alpha}$ emitted from the positions of $\rho=0.01$ (solid line), $\rho=0.24$ (dashed line) and $\rho=0.43$ (dotted line), respectively. The emissivities are normalized to 1.0 at the maximum values. Central electron temperature obtained from Thomson scattering is also plotted with solid circles.

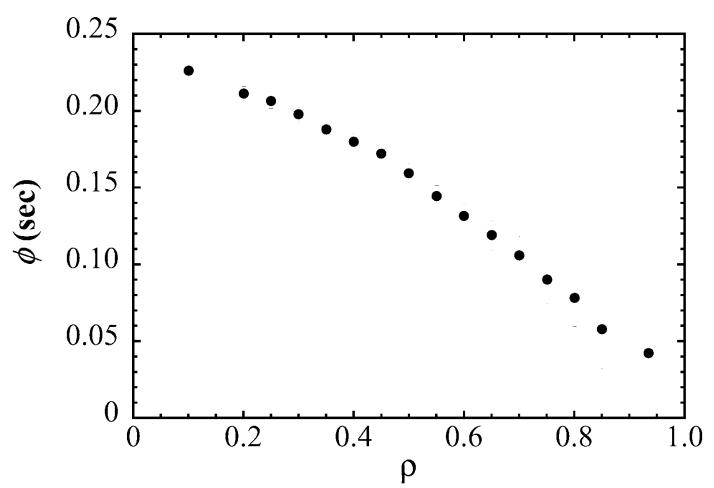

Fig. 4 Radial profile of phase shift estimated from the time evolution of the emissivity profiles as a function of normalized radius. The argon puff is done at $\phi=0$

the Ar- $\mathrm{K}_{\alpha}$ emissions between the Ar-gas-puff timing and the signal peak becomes longer when the observed lineof-sight is shifted to plasma center. Decay time of the Ar$\mathrm{K}_{\alpha}$ emissivity is not so different for all signal channels, as typically estimated to be $3 \mathrm{~s}$ in the figure. Then, the dependence of the time evolution on the radial location is ignored except for the phase shift. This fact gives important information that the continuity equation can be solved with the phase shift.

Figure 4 shows the phase-shift profile quantitatively estimated from the present experimental results. The profile is evaluated at half value of the maximum emissivity, i.e., at 0.5 in Fig. 3. The vertical axis corresponds to the time duration which the Ar requires for reaching the indicated radial position, e.g., $0.225 \mathrm{~s}$ at $\rho=0.1$. The phase shift continuously increases from the plasma edge to the plasma center. The accuracy of the phase-shift profile seems to be enough high to obtain the derivative.

\section{Analysis of Ar-K $K_{\alpha}$ Emissivity}

In the present research the transport coefficients of argon have been derived from a continuity equation which is described by

$$
-\frac{\partial}{\partial t} n(\vec{r}, t)=\nabla \cdot \vec{\Gamma}(\vec{r}),
$$

where $n(\vec{r}, t)$ and $\Gamma(\vec{r})$ are an impurity density and an impurity flux, respectively. In the equation the density and the flux are summed over all charge states of argon. The source terms of each ionized state is assumed to cancel out. The neutral argon puff as the source term is initially localized at the plasma edge.

It is assumed that the impurity flux is expressed by the summation of a diffusion term and a convective term as follows;

$$
\vec{\Gamma}(\vec{r}) \equiv-D(\vec{r}) \nabla n(\vec{r})+\vec{V}(\vec{r}) n(\vec{r}),
$$

where $D(\vec{r})$ and $V(\vec{r})$ are the diffusion coefficient and the convective velocity, respectively. The experimental result shown in Fig. 3 makes it possible that the impurity density is expressed by following equation;

$$
n(\rho, t)=A(\rho) f(t-\phi(\rho)) .
$$

With an assumption of

$$
f(t) \equiv e^{\left(\frac{1}{\tau}+i \omega\right) t},
$$

it is possible to solve Eq. (7) as follows;

$$
\begin{aligned}
D(\rho)= & -a^{2}\left(\rho A(\rho) \frac{\partial}{\partial \rho} \phi(\rho)\right)^{-1} \\
& \times e^{-\frac{\phi(\rho)}{\tau}}\left[\frac{Y_{0}}{\tau}+\left(1-\frac{1}{\tau} \phi(\rho)\right) X_{0}\right],
\end{aligned}
$$

where $a, A(\rho), \omega, \tau$ and $\phi(\rho)$ denote the averaged plasma radius, the impurity density profile, frequency, the decay time and the phase-shift profile, respectively [9]. In Eq. (11) the frequency is assumed to be infinitesimal, since the argon puff is treated to be an ideal single pulse. The sign $X_{0}$ and $Y_{0}$ are defined by

$$
\begin{aligned}
& X_{0} \equiv \int_{0}^{\rho} d \zeta \zeta A(\zeta) e^{\frac{\phi(\zeta)}{\tau}} \text { and } \\
& Y_{0} \equiv \int_{0}^{\rho} d \zeta \zeta \phi(\zeta) A(\zeta) e^{\frac{\phi(\zeta)}{\tau}},
\end{aligned}
$$

respectively. The convective velocity is also solved as

$$
\begin{aligned}
V(\rho)= & \frac{1}{a} e^{-2 \frac{\phi(\rho)}{\tau}} D(\rho) \frac{\partial}{\partial \rho} \ln A(\rho) \\
& +\frac{a}{\rho} A(\rho)^{-1} e^{-\frac{\phi(\rho)}{\tau}} \frac{1}{\tau^{2}}\left[\phi(\rho) X_{0}-Y_{0}\right] .
\end{aligned}
$$

The diffusion coefficient at the plasma center is approximately obtained from only the phase-shift profile and the averaged plasma radius using the following equation;

$$
\lim _{\rho \rightarrow 0} D(\rho)=-\frac{a^{2}}{2}\left(\left.\frac{\partial^{2}}{\partial \rho^{2}} \phi(\rho)\right|_{\rho=0}\right)^{-1} .
$$


This fact is the most favorite advantage in the present research, since the $\mathrm{K}_{\alpha}$ emissivity is generally too much sensitive to the electron temperature in order to estimate the accurate impurity density.

\section{Results of Analysis and Discussions}

Figure 5 shows the radial profile of the diffusion coefficient derived from the phase-shift profile of argon using Eq. (11). It is assumed that the density profile of argon has a weak dependence on the normalized radius as well as the metallic impurity case [6]. As a result, the diffusion coefficient increases from 0.2 to $0.8 \mathrm{~m}^{2} / \mathrm{s}$ in a region smaller than $\rho=0.8$. However, the profile approximately constant in a region between $\rho=0.3$ and $\rho=0.5$. The diffusion coefficient at the plasma center is estimated to be $0.15 \mathrm{~m}^{2} / \mathrm{s}$ using Eq. (15). Figure 6 shows the radial profile of the convective velocity obtained using Eq. (14). The direction of the velocity is inward. The obtained profile is far from the linear relation as a traditional assumption. The convective velocity becomes parabolically large negative with an increasing of $\rho$.

In the analysis the qualitative profile of the diffusion coefficient and convective velocity can individually evaluated. In this case, however, the counting rate of the $\mathrm{x}$ ray emissions must be enough high to obtain the accurate derivative of the profile as included in Eq.(11). Consequently, it is confirmed that the precise phase shift can be obtained with the assembly because of its high brightness.

From the analysis we see two parts of the impurity transport in LHD NBI discharges. In the outer region of the plasma $(0.5<\rho)$ the diffusion coefficient is larger and linearly increases. The convective velocity is also a large negative value. In the inner region of the plasma $(\rho<0.5)$ the diffusion coefficient decreases with a decrease of the normalized radius. The convective velocity becomes also small in the plasma center. These results suggest that the argon ions rapidly penetrate into the outer region of the plasma from the plasma edge, but can not easily go into the plasma center. However, if the argon ions reach the plasma center, they stay long time in the plasma core as seen in Fig. 3.

\section{Summary}

In LHD time-resolved radial profiles of $\operatorname{argon} \mathrm{K}_{\alpha}$ lines have been obtained using an x-ray PHA diagnostic assembly equipped with conventional semiconductor detectors. Especially, the phase-shift profile has been successfully obtained with the assembly. From the experimental results, the radial profiles of the diffusion coefficient and the convective velocity have been individually derived. The dif-

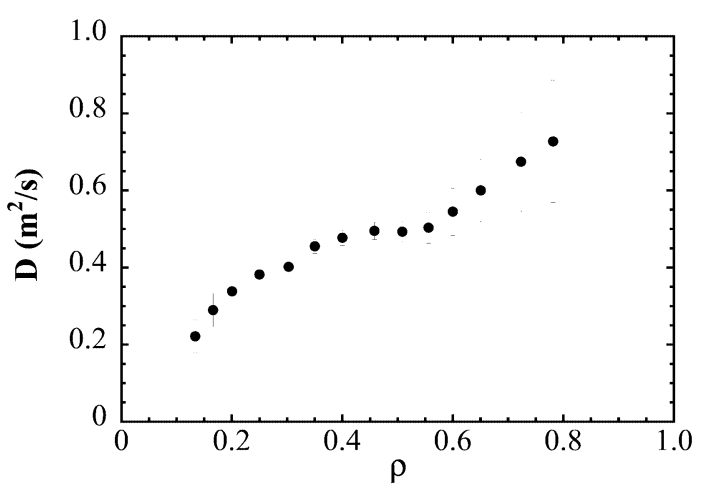

Fig. 5 Radial profile of Diffusion coefficient estimated from the experimental results using Eq. (11)

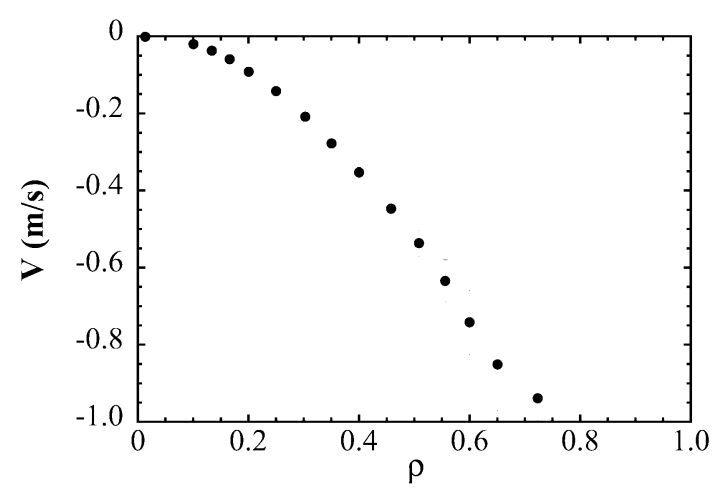

Fig. 6 Radial profile of convective velocity with inward direction.

fusion coefficient increases from $0.15 \mathrm{~m}^{2} / \mathrm{s}$ at the plasma center to $0.8 \mathrm{~m}^{2} / \mathrm{s}$ at outer region of the plasma. The convective velocity is inward and takes a large negative value in the outer region of the plasma.

\section{Acknowledgements}

This work is supported by the LHD project budget (NIFS05ULHH505).

[1] S. Muto et al., Rev. Sci. Instrum. 74, 1993 (2003).

[2] R. Gianalla et al., Nucl. Fusion 34, 1185 (1994).

[3] S. Muto et al., Fusion Eng. Des. 34-35, 205 (1997).

[4] H. Nozato, S. Morita et al., Phys. Plasmas 11, 1920 (2004).

[5] H. Nozato, S. Morita et al., Phys. Plasmas 13, 092502 (2006).

[6] S. Muto et al., 28 th EPS conference Controlled Fusion and Plasma Physics P1.108 (2001).

[7] S. Muto et al., Rev. Sci. Instrum. 72, 1206 (2001).

[8] S. Muto et al., J. Plasma Fusion Res. SERIES 7, 27 (2006).

[9] H. Takenaga et al., J. Plasma Fusion Res. 75, 952 (1999). 